\title{
Fisiopatología de la hepatitis C y diabetes mellitus. Hacia la cura de dos epidemias en el siglo XXI
}

\section{Pathophysiology of Hepatitis C and Diabetes Mellitus: Towards the cure of two epidemics in the $21^{\text {st }}$ century}

\author{
Harold Muñoz Díaz, ${ }^{1}$ Adán Lúquez Mindiola, ${ }^{2 *}$ Andrés Gómez Aldana. ${ }^{3}$
}

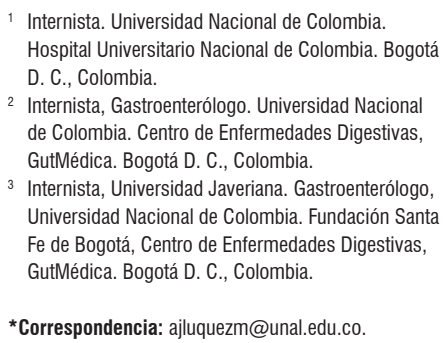

2 Internista, Gastroenterólogo. Universidad Nacional de Colombia. Centro de Enfermedades Digestivas, GutMédica. Bogotá D. C., Colombia.

3 Internista, Universidad Javeriana. Gastroenterólogo, Universidad Nacional de Colombia. Fundación Santa Fe de Bogotá, Centro de Enfermedades Digestivas, GutMédica. Bogotá D. C., Colombia.

*Correspondencia: ajluquezm@unal.edu.co.

\begin{abstract}
Resumen
La infección crónica por virus de la hepatitis $\mathrm{C}(\mathrm{VHC})$ y la diabetes mellitus (DM) son dos problemas de salud pública que impactan los sistemas de salud, con una alta carga económica global. La infección por VHC produce manifestaciones hepáticas tales como hepatitis, cirrosis y carcinoma hepatocelular; asimismo, se ha involucrado en la patogénesis de manifestaciones extrahepáticas, entre las cuales se ha asociado con alteraciones metabólicas como la DM. Estudios longitudinales y transversales han reportado mayor incidencia y prevalencia de DM en pacientes con infección crónica por VHC. La DM acelera la progresión histológica y clínica en pacientes con infección crónica por VHC y las complicaciones cardiovasculares. Recientemente se ha avanzado en el tratamiento y la introducción de nuevos medicamentos como los antivirales de acción directa, que mejoran el control glucémico en estos pacientes.
\end{abstract}

\author{
Palabras clave \\ Hepatitis C, hepatitis C crónica, diabetes mellitus.
}

\begin{abstract}
Chronic hepatitis $\mathrm{C}$ virus (HCV) and diabetes mellitus (DM) are two public health problems that impact health care systems with overall high costs. HCV infections cause liver manifestations such as hepatitis, cirrhosis and hepatocellular carcinoma. They have also been involved in the pathogenesis of extrahepatic manifestations among which are metabolic disorders such as DM. Longitudinal and cross-sectional studies have reported a higher incidence and prevalence of DM in patients with chronic HCV infections. DM accelerates histological and clinical progression of chronic HCV infections and leads to cardiovascular complications. Recently, progress has been made in treatment with the introduction of new medications such as direct-acting antiviral drugs that improve glycemic control in these patients.
\end{abstract}

\section{Keywords}

Hepatitis C, chronic hepatitis C, diabetes mellitus.

\section{INTRODUCCIÓN}

La infección por el virus de la hepatitis C (VHC) y la diabetes mellitus (DM) son dos de los principales problemas en salud pública a nivel mundial que impactan los sistemas de salud y tienen una carga económica global alta en los sistemas financieros, principalmente en los países en vías de desarrollo $(1,2)$.
En la actualidad, se define la DM como un grupo de enfermedades metabólicas cuya característica en común es la hiperglucemia producida por un déficit en la secreción o acción de la insulina, o por ambos (3). La hiperglucemia crónica en la DM se ha asociado a largo plazo con daño, disfunción y falla de varios órganos, principalmente ojos, riñones, nervios, corazón y vasos sanguíneos; lo cual afecta la calidad de vida y aumenta la mortalidad $(3,4)$. 
La DM puede ser clasificada dentro de dos grandes tipos: tipo 1 (DM1) y tipo 2 (DM2). La DM1 corresponde al 5 $\%-10 \%$ de la DM. Se caracteriza por la destrucción de las células $\beta$ del páncreas, que conlleva a un déficit absoluto de insulina, y se debe a un mecanismo autoinmune. Se asocia con el haplotipo HLA-DR/DQ. Los marcadores de destrucción autoinmunitaria son positivos y se encuentran anticuerpos contra las células del islote (ICA) u otros autoanticuerpos contra la descarboxilasa del ácido glutámico (anti-GAD), anticuerpos antiinsulínicos (anti-IA) y anticuerpos anti-tirosina fosfatasa (IA-2, IA-2 beta) $(5,6)$. La DM2 afecta al $90 \%-95 \%$ de los pacientes con DM. Se caracteriza por la resistencia a la insulina y el déficit relativo de esta, en mayor o menor grado; además, puede presentar un aumento de la producción de glucosa hepática. Probablemente haya diferentes causas que originen este tipo de DM, pero la etiología específica no se conoce exactamente (7).

La infección crónica por el VHC tiene una prevalencia entre 1,2 \%-3,8 \% según la región geográfica (8); aproximadamente, 130-175 millones de personas están actualmente infectadas, una población adicional de 3-4 millones serán infectadas cada año y 350 mil personas mueren por año (9). Es la principal causa de trasplante hepático en países desarrollados y la principal causa de morbilidad y mortalidad relacionada con el hígado $(10,11)$.

El VHC es una causa frecuente de enfermedades hepáticas crónicas, incluidas la hepatitis, cirrosis y carcinoma hepatocelular (CHC); asimismo, se ha involucrado en la patogénesis de varias enfermedades autoinmunitarias y reumatológicas como artritis, vasculitis, síndrome sicca, porfiria cutánea tarda, liquen plano, nefropatías, enfermedades tiroideas y fibrosis pulmonar; y en el desarrollo de desórdenes linfoproliferativos de células $\beta(12,13)$. Adicionalmente, se ha asociado con varias manifestaciones extrahepáticas, incluidas las alteraciones metabólicas, dentro de las que se encuentra la DM (14).

El objetivo de este trabajo es revisar la evidencia que asocia la infección crónica por VHC y la DM en diferentes campos: epidemiología, patogénesis, cuadro clínico, tratamiento y prevención.

\section{METODOLOGíA}

Para la realización del presente trabajo se realizó una búsqueda de la literatura utilizando los siguientes términos $\mathrm{MeSH}$ y palabras clave en la estrategia de búsqueda: hepatitis $C$, hepatitis $C$ chronic, diabetes mellitus, epidemiology, physiopathology, diagnosis, therapeutics, antiviral agents.

La búsqueda se limitó a estudios realizados en humanos, en inglés y español, publicados desde la descripción inicial de la asociación en 1994 hasta noviembre de 2018. Las bases de datos electrónicas investigadas fueron Cochrane, Central de Ensayos Controlados, Medline, Embase y Science Citation Index. Adicionalmente, se realizaron búsquedas manuales. Se eligieron las publicaciones más relevantes según los autores.

\section{GENERALIDADES}

El hígado juega un papel importante en el metabolismo de los carbohidratos. Las enfermedades hepáticas crónicas presentan con alta prevalencia alteraciones en la homeostasis de la glucosa, intolerancia a los hidratos de carbono y resistencia a la insulina, lo cual puede eventualmente llevar a DM (Figura 1) (15-17). Asimismo, la elevación moderada y asintomática de aminotransferasas es frecuente en pacientes con DM, especialmente aquellos con DM2, y se ha relacionado con infiltración grasa del hígado (18).

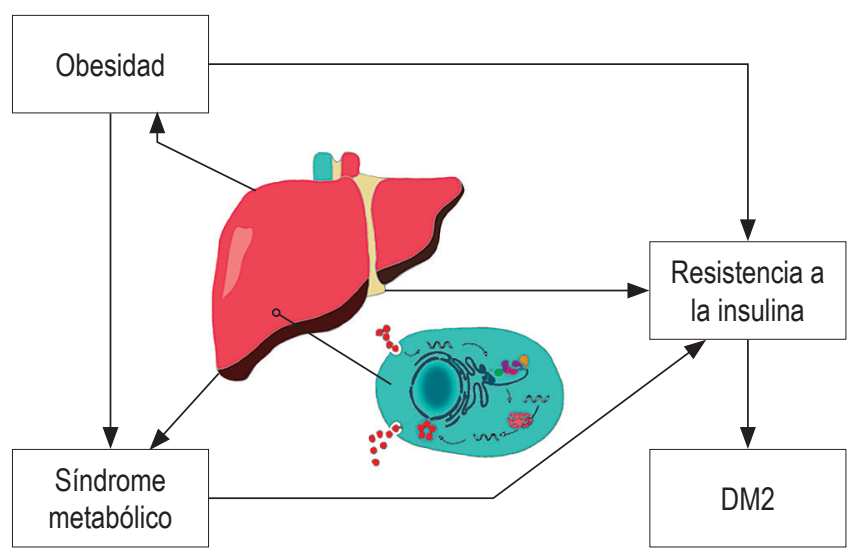

Figura 1. Relación entre VHC y anormalidades metabólicas.

La progresión de la fibrosis hepática es responsable del desarrollo de resistencia a la insulina y DM2; sin embargo, la DM también puede ocurrir en etapas tempranas de la enfermedad hepática $(19,20)$. Inicialmente, se consideraba el daño a largo plazo del hepatocito, como causa de las alteraciones en la homeostasis de la glucosa, pero diferentes estudios han evidenciado que los pacientes con otras patologías hepáticas crónicas como la infección por virus de la hepatitis B (VHB) tienen baja prevalencia de DM, en comparación con pacientes con hepatitis C crónica (21-24).

La prevalencia de anticuerpos VHC en la población con DM2 varía entre $1,78 \%$ y $12,1 \%(25,26)$. Estudios de corte transversal y con un grupo control de individuos no diabéticos establecieron una prevalencia más alta de anticuerpos VHC en pacientes con DM2 que en la población general (27-30). Contrario a lo evidenciado en DM2, la prevalencia de anticuerpos VHC en sujetos con DM1 no excede la prevalencia esperada en la población general (31). El estu- 
dio NHANES III encontró en personas mayores de 40 años con infección por VHC tres veces de probabilidad de tener DM2 con respecto a aquellos no infectados, y no se detectó una asociación con la DM1 (32). Otro gran estudio basado en la comunidad estableció que la positividad de anticuerpos VHC estuvo fuertemente asociada con DM2 entre los 39 a 49 años de edad (33).

Cuando los pacientes infectados por VHC y cirrosis son evaluados, la prevalencia de DM2 es más alta que la reportada en pacientes con hepatitis crónica, y varía de $19,6 \%$ a 50 $\%$ (34). Un estudio prospectivo que comparó 50 pacientes con cirrosis debido a infección por VHC y 50 pacientes con cirrosis sin infección por VHC encontró una mayor prevalencia de $\mathrm{DM}$ en los pacientes infectados con $\mathrm{VHC}$ y valores más altos de insulinemia basal; parecería que la presencia de enfermedad hepática avanzada es un factor diabetogénico fuerte, igual que la infección por VHC en sí misma (34).

La consecuencia clínica de la alta prevalencia de infección por $\mathrm{VHC}$ en DM2 es que las elevaciones leves de aminotransferasas séricas no deben atribuirse automáticamente a enfermedad por hígado graso no alcohólico y, por ello, las pruebas para la infección por este virus en pacientes diabéticos con perfil hepático alterado deben ser mandatorias (35).

Hay información insuficiente acerca de la duración de la infección por VHC y DM que permita evaluar su relación temporal. Un estudio reportó que todos los pacientes con infección de VHC y DM2 tenían historia previa de transfusión de hemoderivados entre 10 y 20 años antes del inicio de la DM (31). En otro estudio se diagnosticó DM2 18 años después de la infección por VHC (36). Cuando la secuencia temporal es caracterizada en pacientes diabéticos VHC positivos, el diagnóstico de la infección precedió a la DM en un $73 \%$ de los casos (37).

En el $52 \%$ de las personas con infección por VHC y DM2 hay factores de riesgo para infección por el virus antes del inicio de la DM, mientras que ninguno tuvo factores de riesgo para infección por el virus después del inicio de la DM (15). La ausencia de cualquier factor epidemiológico para infección por VHC entre los pacientes diabéticos y la evidencia que sugiere que la infección precede a la DM soporta la idea de que el virus puede causar o predisponer a las personas infectadas por VHC para que desarrollen DM; sin embargo, las conclusiones definitivas se deben realizar con estudios prospectivos. La edad, obesidad, historia familiar de DM, origen afroamericano y coinfección de VHC y virus de inmunodeficiencia humana (VIH) son factores de riesgo asociados con el desarrollo de DM entre personas infectadas con VHC $(38,39)$. Por el contrario, la relación entre DM2 y los genotipos del VHC permanece controversial con respecto a la existencia de algún genotipo específico que predisponga o proteja del desarrollo de DM en pacientes infectados (40).
La diabetes mellitus postrasplante (DMPT) es una condición médica que surge durante el seguimiento de receptores de trasplante renal y hepático, la cual ha incrementado su incidencia en las últimas décadas (41). Existen factores de riesgo independientes para su desarrollo tales como agentes inmunosupresores para prevenir y tratar el rechazo, origen del donante y los relacionados con el receptor (42). $\mathrm{La}$ infección crónica por VHC es una de las indicaciones actuales que llevan a trasplante hepático ortotópico. La prevalencia de DMPT en receptores de trasplante hepático infectados con VHC está entre $40 \%-64 \%$, significativamente más alta que la prevalencia reportada en pacientes trasplantados por otras causas de insuficiencia hepática. Además, se ha establecido que el VHC es un factor de riesgo independiente para el desarrollo de DMPT (42). La infección por VHC puede alcanzar una incidencia de $50 \%$ en pacientes con enfermedad renal terminal y se ha identificado como un factor de riesgo independiente para el desarrollo de DMPT después de un trasplante renal (43). Todos estos datos refuerzan la hipótesis de que el VHC es más una causa que una consecuencia de la DM. Adicionalmente, la relación entre VHC y DM puede contribuir sustancialmente al papel perjudicial del virus en la supervivencia del paciente y el injerto después del trasplante hepático o renal (43).

Se ha explorado el vínculo entre la infección por VHC y la DM mediante la evaluación de la prevalencia no solo de la DM, sino también de la glucosa alterada en ayunas (GAA). En una cohorte de pacientes con hepatitis crónica por VHC se observó un incremento de casi 3 veces la prevalencia de anormalidades en la glucosa en comparación con los pacientes negativos de VHC o con otras hepatopatías ( $32 \%$ frente a $12 \%)$. Entre los pacientes con infección por VHC se encontró una mayor prevalencia de DM y GAA ( $17 \%$ frente a $7 \%$, y $15 \%$ frente a $5 \%$, respectivamente). No se observaron diferencias en pacientes cirróticos con o sin infección por VHC. Estos hallazgos sugieren que la conexión genuina entre la infección del VHC y la DM se inicia en las etapas tempranas de la enfermedad hepática (40).

La alta prevalencia de alteraciones en el metabolismo de la glucosa encontradas en pacientes con infección por VHC sugiere que deben considerarse un grupo de alto riesgo para desarrollar DM y GAA; y por tanto, se debería realizar tamización para DM. Lecube y colaboradores realizaron una prueba de tolerancia oral a la glucosa (PTOG) en 50 pacientes positivos de VHC y 50 pacientes negativos de VHC con hepatitis crónica en quienes la DM no se había diagnosticado. Ambos grupos se emparejaron por edad, índice de masa corporal (IMC) y sexo. La PTOG permitió diagnosticar 18 $\%$ de casos nuevos de DM y $30 \%$ de GAA en pacientes positivos de VHC en comparación con los pacientes negativos de VHC (4 \% y $18 \%$, respectivamente) (44). 
En la Tabla 1 se definen los criterios para el diagnóstico de DM según la Asociación Americana de Diabetes (ADA) (45).

Tabla 1. Criterios para el diagnóstico de DM

\begin{tabular}{lc}
\hline \multicolumn{1}{c}{ Criterio } & Cantidad \\
\hline Glucemia al azar* & $\geq 200 \mathrm{mg} / \mathrm{dL}$ \\
Glucemia en ayunas* & $\geq 126 \mathrm{mg} / \mathrm{dL}$ \\
PTOG* $^{*}$ & $\geq 200 \mathrm{mg} / \mathrm{dL}$ \\
HBA $_{1 C}$ & $\geq 6,5 \%$ \\
\hline
\end{tabular}

*El diagnóstico requiere dos resultados anormales de la misma muestra o en dos muestras separadas. $\mathrm{HBA}_{1 \mathrm{C}}$ : hemoglobina glicosilada; PTOG: prueba de tolerancia oral a la glucosa.

\section{MECANISMOS PATOGÉNICOS INVOLUCRADOS EN LA ACCIÓN DIABETOGÉNICA DEL VHC}

\section{Efectos del VHC y resistencia a la insulina}

El VHC es hepatotropo y no citopático; su genoma se ha identificado en tejidos más allá del hígado, incluidas las células acinares pancreáticas y células epiteliales del ducto pancreático (46). Estudios post mortem revelaron que el VHC se replica en el páncreas y modelos animales sugieren un efecto directo de la infección en la resistencia a la insulina a nivel hepático (47).

El virus tiene un genoma de ácido ribonucleico (ARN) de 9,6 Kb, que codifica aproximadamente 3010 aminoácidos y son transportados en proteínas estructurales (core, E1, E2) y proteínas no estructurales (NS3-NS5B). Estas proteínas juegan un papel importante en el desarrollo de resistencia a la insulina y estrés oxidativo por medio de especies reactivas de oxígeno a nivel celular (48). La proteína core, sola o en combinación con otras proteínas virales, incrementa la fosforilación del sustrato del receptor de insulina tipo 1 (SRI1), el cual es la base de la resistencia a la insulina (49). El SRI-1 fosforilado activa la fosfatidilinositol 3 cinasa (PI3K), y la activación de PI3K y Akt es esencial para muchos de los efectos metabólicos de la insulina (Figura 2) (50); por ello, los defectos a nivel de la asociación de PI3K con SRI-1 y la pérdida de activación de la PI3K pueden contribuir a la resistencia a la insulina y prevalencia incrementada de DM en sujetos infectados con VHC. Este mecanismo, por último, promueve la translocación del transportador 4 de glucosa (GLUT4) a la membrana plasmática para mejorar la captación de glucosa (51). Además, la proteína core puede directamente activar los inhibidores de la señalización de la insulina, tales como mTOR (blanco de rapamicina en células de mamífero), SOCS-3 (supresor de señalización de citocinas) y JNK (cinasa c-Jun $\mathrm{N}$-terminal) (52). Adicionalmente, el VHC aumenta el estrés en el retí- culo endoplasmático, lo cual activa la proteína fosfatasa $2 \mathrm{~A}$ (PP2A), que inhibe dos reguladores claves en la gluconeogénesis: proteína cinasa activada por el monofosfato de adenosina (AMPK) y Akt (52).

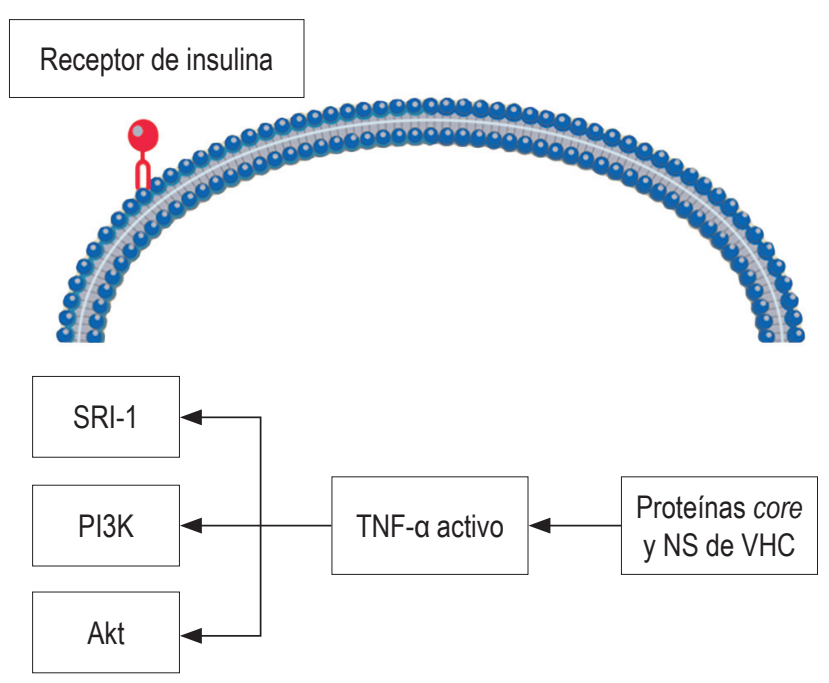

Figura 2. Mecanismo por el cual el VHC interfiere en la señalización de la insulina. TNF- $\alpha$ : factor de necrosis tumoral alfa.

Reciente evidencia soporta la existencia de un componente extrahepático de resistencia a la insulina inducida por el virus; de este modo, la patogénesis molecular de las alteraciones del metabolismo de glucosa observadas en la infección por VHC son mucho más complejas de lo que se creía $(51)$.

\section{Citocinas proinflamatorias}

Las estrategias virales de evasión inmune innatas y las determinantes genéticas humanas son la base de la transición de infección aguda hacia la persistencia viral e infección crónica. Los factores genéticos del huésped pueden influenciar el resultado de la infección y la respuesta a la terapia antiviral. Estudios recientes revelan una compleja interacción entre el contexto genético del paciente con factores virales y del huésped relacionados con el desencadenante inmune innato, que dicta el control de la infección e inmunidad del VHC (53).

Más allá de los efectos directos del VHC en SRI-1/PI3K, la proteína core puede inducir resistencia a la insulina indirectamente por medio de la estimulación de la secreción de citocinas proinflamatorias. En pacientes con VHC crónica, la inflamación inducida por el virus produce hipersecreción de citocinas proinflamatorias resistentes a la insulina, como la interleucina 6 (IL-6) y el TNF- $\alpha(52,54,55)$. Las citocinas proinflamatorias también regulan los supresores de proteínas de la señal de las citocinas como parte de un circuito de retroalimentación negativo al atenuar la 
señal de estas (56). Este fenómeno puede contribuir a un incremento en la gluconeogénesis debido a la pérdida de la inhibición mediada por Akt de la expresión del gen de la fosfoenolpiruvato carboxicinasa (FEPCK). En este contexto, es interesante anotar que la leptina puede modular la acción de la insulina en las células hepáticas por antagonizar la fosforilación de SRI-1 estimulada por insulina, incrementando la expresión del gen FEPCK y disminuyendo la expresión de la glucocinasa, lo cual resulta en un aumento de la gluconeogénesis (57). Con el incremento en la gluconeogénesis, después de la infección por VHC, hay mayor producción y acumulación de lípidos mediada por la inhibición de la AMPK (58). El efecto estimulante de lipólisis por el TNF- $\alpha$ lleva a niveles séricos elevados de ácidos grasos libres, los cuales reducen la sensibilidad a la insulina $(59,60)$.

Las citocinas son mediadores intercelulares involucrados en el control viral y el daño hepático inducido por el VHC. La compleja red de citocinas que opera durante la infección inicial permite el desarrollo coordinado y efectivo de las respuestas inmunes innata y adaptativa; sin embargo, el virus interfiere con las citocinas en varios niveles y escapa a la respuesta inmunitaria induciendo un perfil $\mathrm{T}_{\mathrm{h}} 2$. La incapacidad para controlar la infección lleva al reclutamiento de infiltrados inflamatorios en el parénquima hepático por interferón gamma (IFN- $\gamma$ ) e induce quimiocinas CXCL9, CXCL10 y CXCL11, lo cual resulta en un daño hepático sostenido y eventualmente cirrosis $(61,62)$. Eslam y colaboradores encontraron polimorfismos en la región IFNL3 (IL-28B) asociados con recuperación espontánea, e inducida por el tratamiento de la infección (63).

Las enfermedades sistémicas extrahepáticas más importantes relacionadas con VHC (crioglobulinemia mixta, desórdenes linfoproliferativos, enfermedades autoinmunitarias de tiroides, DM2) están asociadas con alteraciones en la regulación compleja de la red citocina/quimiocina, involucrando quimiocinas proinflamatorias y respuesta Th1 $(61,62)$.

\section{Infección por VHC y DM1}

Distintos mecanismos se han postulado. Así como el VHC puede infectar tejidos extrahepáticos, la participación de este en el inicio de DM1 aún no se ha aclarado (64). Más allá de los mecanismos directos no demostrados, la infección por VHC inicia o acelera una reacción inmunitaria contra las células $\beta$. La infección de células $\beta$ por VHC puede regular la expresión y secreción del gen CXCL10 y reclutamiento de linfocitos Th 1 , que secretan IFN- $\gamma$ y TNF- $\alpha$, los cuales inducen la secreción de CXCL10 por células $\beta$ y de este modo perpetúan la cascada inmunitaria; dicha cascada puede llevar a disfunción de células $\beta$ en sujetos predis- puestos genéticamente (Figura 3) (65). Adicionalmente, se sugiere el mimetismo molecular con un desencadenante de autoinmunidad relacionada con el VHC, la descarboxilasa del ácido glutámico (GAD) comparte una secuencia de 65 aminoácidos similar con regiones antigénicas de la poliproteína del virus (66). Otra posibilidad es la inducción de anticuerpos reactivos contra GAD y el desarrollo de DM mediada por interleucina 18 (IL-18) y otras citocinas proinflamatorias (67). La IL-18 juega un papel patogénico en DM1 porque está involucrada en la aceleración del desarrollo de la enfermedad manifiesta, puede inducir respuesta Th1 y Th2 dependiendo de las citocinas circundantes, además de jugar un rol patogénico en distintas enfermedades incluida la insuficiencia hepática aguda (67, 68). Otras citocinas proinflamatorias, tales como, TNF- $\alpha$ e IL-1B, que están elevadas en pacientes con hepatitis aguda, pueden también inducir diabetes autoinmune (69).

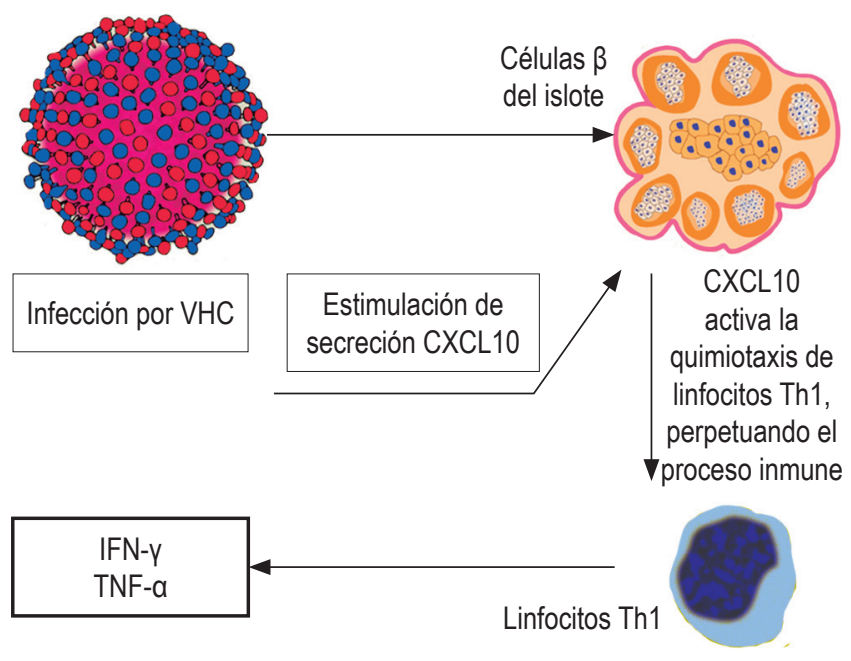

Figura 3. Regulación potencial de las manifestaciones endocrinas del VHC en las células $\beta$ del islote pancreático.

\section{Sobrecarga de hierro}

Altas concentraciones de ferritina están asociadas con resistencia a la insulina y con riesgo incrementado de DM2 en personas sanas. En los pacientes infectados con VHC se ha reportado el incremento de los niveles de ferritina (70). La ferritina es un reactante de fase aguda que puede estar alterado por procesos inflamatorios, y la evidencia sugiere que en DM2 existe un bajo grado de inflamación. Los pacientes con infección por VHC sin DM no tienen niveles altos de ferritina, por lo cual se sugiere que la DM, y no la infección por $\mathrm{VHC}$, es un factor de riesgo para concentraciones altas de hierro; sin embargo, hacen falta más estudios prospectivos que evalúen esta relación (70). Adicionalmente, los depósitos de hierro hepáticos pueden causar resistencia a la 
insulina al interferir con la capacidad de la insulina de suprimir la producción de glucosa hepática (70).

\section{Esteatosis hepática}

Es más frecuente en infección por VHC que en infección por VHB y ocurre en más del $50 \%$ de pacientes con VHC crónica (71). La esteatosis leve se asocia con IMC elevado y obesidad visceral, mientras que la esteatosis moderada a grave es causada directamente por el virus (52). Las diferencias genotípicas en términos de progresión de las alteraciones hepáticas también se han descrito. La esteatosis en infección por VHC genotipo 4 es una expresión de síndrome metabólico causado por la activación de mecanismos proinflamatorios, así como la obesidad y la resistencia a la insulina. El grado de esteatosis en este genotipo es independiente de la carga viral y la terapia antiviral no la mejora (72). El genotipo 3a se relaciona principalmente con esteatosis grave (52).

La esteatosis hepática puede contribuir a DM asociada con el VHC por daño en la capacidad de la insulina de disminuir la gluconeogénesis y favorecer la fibrosis hepática (71).

\section{DM en infectados con VHC tratados con interferón alfa (IFN- $\alpha)$}

Algunos estudios han mostrado una alta prevalencia de marcadores de autoinmunidad pancreática en pacientes con infección por VHC durante o después de la terapia con IFN- $\alpha$, probablemente debido a los efectos inmunoestimuladores de estas citocinas (73). El IFN- $\alpha$ tiene actividad antiviral, antiproliferativa e inmunomoduladora; en individuos predispuestos, puede inducir un proceso diabetogénico. Por esta razón, los autoanticuerpos de células del islote y anti-GAD deben ser investigados antes y durante el tratamiento para identificar individuos con alto riesgo de desarrollar DM1 (73). Algunos pacientes pueden desarrollar autoinmunidad pancreática de novo y estar en riesgo de desarrollar DM. Los pacientes que son inicialmente positivos para autoanticuerpos organoespecíficos (especialmente autoanticuerpos específicos de páncreas y tiroides) y aquellos quienes hacen seroconversión están en alto riesgo de desarrollar enfermedad autoinmunitaria clínica después del tratamiento (74). El IFN- $\alpha$ incrementa la expresión de antígenos HLA clase I, activa las células NK (natural killer) y linfocitos T, y puede ser un importante cofactor en el desarrollo de una reacción inmunitaria Th1 (75). La suspensión oportuna de la terapia es raramente acompañada por regresión clínica de la $\mathrm{DM}(74)$.

\section{Cáncer en infección por VHC y DM}

La principal característica de los pacientes diabéticos es la resistencia a la insulina, la cual es crucial para la progresión de la fibrosis y su impacto negativo en la respuesta al tratamiento con antivirales en sujetos con VHC crónica (76). La reducida sensibilidad a la insulina es la base de la hiperinsulinemia compensatoria y los niveles elevados de factor de crecimiento similar a la insulina tipo 1 (IGF-1), el cual estimula la proliferación celular e inhibe la apoptosis. Este fenómeno tiene fuertes efectos mitogénicos en una amplia variedad de líneas celulares cancerígenas (77). Al mismo tiempo, la insulina activa el receptor de IGF-1, que tiene un efecto promotor de crecimiento que incluye la modulación de la progresión del ciclo celular. El exceso de insulina puede llevar indirectamente al desarrollo de cáncer por la regulación del nivel de proteína 1 ligada a IGF, lo que incrementa el nivel y biodisponibilidad de IGF-1 circulante. La obesidad e inactividad física también causan hiperinsulinemia y por esto también se asocia con progresión acelerada de cáncer (78).

La VHC crónica es una forma progresiva de enfermedad hepática que lleva a cirrosis y CHC (13). Los pacientes con infección por VHC diabéticos tienen un riesgo incrementado de $\mathrm{CHC}$ en comparación con individuos no diabéticos, por lo que la DM parece tener un impacto selectivo en el desarrollo del CHC (79). Es así como los pacientes con DM2 en quienes se logra un buen control glucémico alcanzando niveles de $\mathrm{HbA}_{1 \mathrm{c}}<7 \%$ se puede reducir el riesgo de $\mathrm{CHC}(80)$.

\section{TRATAMIENTO Y PREVENCIÓN}

Estudios clínicos en pacientes con infección por VHC han reportado mejoría en el control glucémico y resistencia a la insulina con los agentes antivirales de acción directa (AAD) en pacientes con o sin DM (81-83). Los pacientes diabéticos quienes reciben $\mathrm{AAD}$ deben ser monitorizados estrictamente para reducir los medicamentos antidiabéticos, principalmente insulina y sulfonilureas, para evitar hipoglucemias (84).

Con la llegada de las terapias para VHC se han encontrado relaciones interesantes, identificando que los primeros tratamientos basados en interferón actuaban como facilitadores en el desarrollo de DM (85). Sin embargo, con las terapias antivirales orales, se ha observado un descenso en la incidencia de DM: en 5127 pacientes tratados para VHC se encontró una incidencia del 6,2\% entre quienes alcanzaron una respuesta virológica sostenida, en contraste con un $21,7 \%$ de los pacientes que tuvieron falla terapéutica después de 3,7 años de seguimiento (Hazard ratio ajustado [aHR] = 0,79; intervalo de confianza [IC] $95 \%$ : 0,65-0,96) (86). Li 
y colaboradores realizaron seguimiento a 1395 pacientes con infección por VHC y DM2 tratados y con respuesta virológica sostenida por un lapso promedio de 2,7 años, y se observó un descenso significativo en las complicaciones como síndrome coronario agudo (Hazard ratio $[\mathrm{HR}]=0,36$; $p<0,001)$ enfermedad renal crónica terminal $(\mathrm{HR}=0,46 ; p$ $<0,001)$, evento cerebrovascular (sub-Hazard ratio [sHR] $=0,34 ; p<0,001)$, y retinopatía $(\mathrm{sHR}=0,24 ; p<0,001)$, al comparar con pacientes no tratados (87).

Se ha podido identificar mejoría en otras variables vinculadas con la $\mathrm{DM}$, como la $\mathrm{HbA}_{1 \mathrm{c}}$, demostrando en estudios recientes que la caída en $\mathrm{HbA}_{1 c}$ fue mayor en aquellos pacientes quienes alcanzaron respuesta virológica sostenida $(0,6 \%$ a $0,98 \%)$ con respecto a quienes fallaron en el tratamiento $(88,89)$, estos beneficios endocrinos proveen una justificación adicional para considerar el tratamiento antiviral en todos los pacientes con infección por VHC y DM (89). Adicionalmente, el tratamiento de la infección por VHC en pacientes con DM podría disminuir la prevalencia de complicaciones, entre ellas, nefrológicas crónicas relacionadas con esta (90).

Diferentes factores pueden alterar esta respuesta, como el genotipo del virus, factores genéticos del hospedero y comorbilidades (91). Algunas investigaciones han reportado que la obesidad y la hipercolesterolemia son factores potenciales que interfieren con la respuesta viral sostenida (92). Esto sugiere opciones terapéuticas adicionales para la infección por VHC que incluyen cambios en la dieta, medicamentos antidiabéticos y estatinas. En el contexto de los antidiabéticos orales no es claro si el mejor enfoque es usar una biguanida, como la metformina (93). Con respecto a las estatinas, estas son capaces de inhibir la replicación del VHC in vitro, pero no in vivo (94).

La relación potencial entre infección por VHC y el desarrollo de DM incrementa la necesidad de implementar medidas de prevención; estas deben ser dirigidas a cambios en el estilo de vida que pueden reducir el riesgo de desarrollar infección por VHC y DM, tamización regular para DM en pacientes con infección por $\mathrm{VHC}$ y análisis de otros factores de riesgo que pueden acelerar la progresión de ambos, tales como obesidad, dislipidemia y consumo de alcohol (95).

Son necesarios estudios adicionales para mejorar las políticas de prevención y fomentar programas adecuados y costo-efectivos para la vigilancia y tratamiento de pacientes diabéticos con VHC crónica, por lo cual se debe implementar un tratamiento multifactorial para curar dos enfermedades: DM y VHC crónica.

\section{CONCLUSIONES}

La infección por VHC y DM son dos desórdenes con un alto impacto en la salud a nivel mundial. Hay alta prevalencia de
DM2 en pacientes infectados por VHC con hepatitis crónica $y$ evidencia creciente que soporta el concepto que esta infección es un factor de riesgo para desarrollar DM o alteraciones en el metabolismo de los hidratos de carbono. Los mecanismos específicos a través de los cuales el VHC está asociado con DM parece involucrar efectos virales directos, resistencia a la insulina, citocinas proinflamatorias, quimiocinas, supresores de señalización de citocinas y otros mecanismos inmunomediados. Estos mecanismos son iniciados en las etapas tempranas de la enfermedad hepática.

La edad, obesidad, historia familiar de DM, origen afroamericano y coinfección VHC-VIH son factores de riesgo asociados con el desarrollo de DM entre personas infectadas con VHC. En estos pacientes se deben realizar estudios para evaluar alteraciones en el metabolismo de los carbohidratos. Se han reportado pocos datos de la asociación de VHC crónica y DM1. La terapia con IFN- $\alpha$ puede estimular autoinmunidad pancreática $y$, en ciertos casos, llevar al desarrollo de DM1. Los pacientes diabéticos con VHC crónica tienen riesgo incrementado de desarrollar cirrosis y CHC en comparación con los pacientes con VHC crónica no diabéticos. El tratamiento con $\mathrm{AAD}$ mejora el control glucémico y la resistencia a la insulina. Se necesitan estudios adicionales para mejorar políticas de prevención $\mathrm{y}$ fomentar programas adecuados y costo-efectivos para el diagnóstico, tratamiento y seguimiento de pacientes diabéticos con VHC crónica.

\section{Agradecimientos}

Ninguno declarado por los autores.

\section{Conflicto de interés}

Ninguno declarado por los autores.

\section{Fuente de financiación}

Ninguna declarada por los autores.

\section{REFERENCIAS}

1. Stepanova M, Younossi ZM. Economic burden of hepatitis C. Clin Liver Dis. 2017;21:579-94. doi: 10.1016/j. cld.2017.03.012.

2. Shaw JE, Sicree RA, Zimmet PZ. Global estimates of the prevalence of diabetes for 2010 and 2030. Diabetes Res Clin Pract. 2010;87:4-14. doi: 10.1016/j.diabres.2009.10.007.

3. Fox CS, Golden SH, Anderson C, Bray GA, Burke LE, de Boer $\mathrm{IH}$, et al. Update on prevention of cardiovascular disease in adults with type 2 diabetes mellitus in light of recent evidence: a scientific statement from the American Heart Association and the American Diabetes Association. 
Diabetes Care. 2015;38:1777-803. doi: 10.2337/dci150012.

4. Vaidya V, Gangan N, Sheehan J. Impact of cardiovascular complications among patients with type 2 diabetes mellitus: a systematic review. Expert Rev Pharmacoecon Outcomes Res. 2015;15:487-97. doi: 10.1586/14737167.2015.1024661.

5. Barbeau WE. What is the key environmental trigger in type 1 diabetes--is it viruses, or wheat gluten, or both? Autoimmun Rev. 2012;12:295-9. doi: 10.1016/j.autrev.2012.05.003.

6. Askenasy EM, Askenasy N. Is autoimmune diabetes caused by aberrant immune activity or defective suppression of physiological self-reactivity? Autoimmun Rev 2013;12:6337. doi: 10.1016/j.autrev.2012.12.004.

7. Ferrannini E. Physiology of glucose homeostasis and insulin therapy in type 1 and type 2 diabetes. Endocrinol Metab Clin North Am. 2012;41:25-39. doi: 10.1016/j.ecl.2012.01.003.

8. Thrift AP, El-Serag HB, Kanwal F. Global epidemiology and burden of HCV infection and HCV-related disease. Nat Rev Gastroenterol Hepatol. 2017;14:122-32. doi: 10.1038/ nrgastro.2016.176.

9. Mohamed AA, Elbedewy TA, El-Serafy M, El-Toukhy N, Ahmed W, Ali El Din Z. Hepatitis C virus: A global view. World J Hepatol. 2015;7:2676-80. doi: 10.4254/wjh. v7.i26.2676.

10. Alter MJ. Epidemiology of hepatitis C virus infection. World J Gastroenterol. 2007;13:2436-41. doi: 10.3748/wjg.v13. i17.2436.

11. Mohd Hanafiah K, Groeger J, Flaxman AD, Wiersma ST. Global epidemiology of hepatitis $C$ virus infection: new estimates of age-specific antibody to HCV seroprevalence. Hepatology. 2013;57:1333-42. doi: 10.1002/hep.26141.

12. Antonelli A, Ferri C, Galeazzi M, Giannitti C, Manno D, Mieli-Vergani G, et al. HCV infection: pathogenesis, clinical manifestations and therapy. Clin Exp Rheumatol. 2008;26(1 Suppl 48): S39-47.

13. Ferri C, Antonelli A, Mascia MT, Sebastiani M, Fallahi P, Ferrari D, et al. HCV-related autoimmune and neoplastic disorders: the HCV syndrome. DigLiver Dis. 2007;39(Suppl 1):S13-21. doi: 10.1016/S1590-8658(07)80005-3.

14. Nocente R, Ceccanti M, Bertazzoni G, Cammarota G, Silveri NG, Gasbarrini G. HCV infection and extrahepatic manifestations. Hepatogastroenterology. 2003;50:1149-54.

15. Mason AL, Lau JY, Hoang N, Qian K, Alexander GJ, Xu L, et al. Association of diabetes mellitus and chronic hepatitis $\mathrm{C}$ virus infection. Hepatology. 1999;29:328-33. doi: 10.1002/ hep.510290235.

16. Weinman SA, Belalcazar LM. Hepatitis C: a metabolic liver disease. Gastroenterology 2004; 126: 917-9. doi: 10.1053/j. gastro.2003.01.001.

17. Bugianesi E, McCullough AJ, Marchesini G. Insulin resistance: a metabolic pathway to chronic liver disease. Hepatology. 2005;42:987-1000. doi: 10.1002/hep.20920.

18. Vozarova B, Stefan N, Lindsay RS, Saremi A, Pratley RE, Bogardus $C$, et al. High alanine aminotransferase is associated with decreased hepatic insulin sensitivity and predicts the development of type 2 diabetes. Diabetes. 2002;51(6):1889-95. doi: 10.2337/diabetes.51.6.1889.

19. Romero-Gomez M. Insulin resistance and hepatitis C. World J Gastroenterol. 2006;12:7075-80. doi: 10.3748/wjg. v12.i44.7075.

20. Petit JM, Bour JB, Galland-Jos C, Minello A, Verges B, Guiguet $M$, et al. Risk factors for diabetes mellitus and early insulin resistance in chronic hepatitis C. J Hepatol. 2001;35:279-83. doi: 10.1016/S0168-8278(01)00143-X.

21. Mehta SH, Brancati FL, Strathdee SA, Pankow JS, Netski $\mathrm{D}$, Coresh J, et al. Hepatitis $\mathrm{C}$ virus infection and incident type 2 diabetes. Hepatology. 2003;38:50-6. doi: 10.1053/ jhep.2003.50291.

22. Huang JF, Dai CY, Hwang SJ, Ho CK, Hsiao PJ, Hsieh MY, et al. Hepatitis $C$ viremia increases the association with type 2 diabetes mellitus in a hepatitis $\mathrm{B}$ and $\mathrm{C}$ endemic area: an epidemiological link with virological implication. Am J Gastroenterol. 2007;102:1237-43. doi: 10.1111/j.15720241.2007.01181.x.

23. Boluda Monzo S, Mesa Manteca J, Obiols Alfonso G, Simo Canonge R. Surface antigen of hepatitis B in diabetes mellitus. Med Clin (Barc). 1989;92:397.

24. Mangia A, Schiavone G, Lezzi G, Marmo R, Bruno F, Villani $\mathrm{MR}$, et al. HCV and diabetes mellitus: evidence for a negative association. Am J Gastroenterol. 1998;93:2363-7. doi: 10.1111/j.1572-0241.1998.00688.x.

25. Ozyilkan E, Erbas T, Simsek H, Telatar F, Kayhan B, Telatar $\mathrm{H}$. Increased prevalence of hepatitis $\mathrm{C}$ virus antibodies in patients with diabetes mellitus. J Intern Med. 1994;235:2834. doi: 10.1111/j.1365-2796.1994.tb01075.x.

26. Gray H, Wreghitt T, Stratton IM, Alexander GJ, Turner RC, O'Rahilly S. High prevalence of hepatitis C infection in Afro-Caribbean patients with type 2 diabetes and abnormal liver function tests. Diabet Med. 1995;12:244-9. doi: 10.1111/j.1464-5491.1995.tb00466.x.

27. Fabiani S, Fallahi P, Ferrari SM, Miccoli M, Antonelli A. Hepatitis $\mathrm{C}$ virus infection and development of type 2 diabetes mellitus: Systematic review and meta-analysis of the literature. Rev Endocr Metab Disord. 2018;19(4):405-20. doi: 10.1007/s11154-017-9440-1.

28. Sotiropoulos A, Peppas TA, Skliros E, Apostolou O, Kotsini V, Pappa SI. Low prevalence of hepatitis C virus infection in Greek diabetic patients. Diabet Med. 1999;16:250-2. doi: 10.1046/j.1464-5491.1999.00009.x.

29. Ryu JK, Lee SB, Hong SJ, Lee S. Association of chronic hepatitis $C$ virus infection and diabetes mellitus in Korean patients. Korean J Intern Med. 2001;16:18-23. doi: 10.3904/kjim.2001.16.1.18.

30. Okan V, Araz M, Aktaran S, Karsligil T, Meram I, Bayraktaroglu Z, et al. Increased frequency of HCV but not HBV infection in type 2 diabetic patients in Turkey. Int J Clin Pract. 2002;56:175-7.

31. Cerutti F, Palomba E, Sacchetti C, Gay V, Versace A, Tovo PA. Anti-HCV antibodies in a population of insulin-dependent diabetic children and adolescents. Diabetes Care. 1999;22:1587-8. doi: 10.2337/diacare.22.9.1587. 
32. Mehta SH, Brancati FL, Sulkowski MS, Strathdee SA, Szklo M, Thomas DL. Prevalence of type 2 diabetes mellitus among persons with hepatitis $\mathrm{C}$ virus infection in the United States. Ann Intern Med. 2000;133:592-9. doi: 10.7326/0003-4819-133-8-200010170-00009.

33. Wang CS, Wang ST, Yao WJ, Chang TT, Chou P. Communitybased study of hepatitis $C$ virus infection and type 2 diabetes: an association affected by age and hepatitis severity status. Am J Epidemiol. 2003;158:1154-60. doi: 10.1093/ aje/kwg259.

34. Garrido Serrano A, Guerrero Igea FJ, Lepe Jimenez JA, Palomo Gil S, Grilo Reina A. Hyperinsulinemia in cirrhotic patients infected with hepatitis $\mathrm{C}$ virus infection. Gastroenterol Hepatol. 2001;24:127-31. doi: 10.1016/ S0210-5705(01)70138-0.

35. Simo R, Hernandez C, Genesca J, Jardi R, Mesa J. High prevalence of hepatitis $\mathrm{C}$ virus infection in diabetic patients. Diabetes Care 1996;19:998-1000. doi: 10.2337/diacare.19.9.998.

36. Grimbert S, Valensi P, Levy-Marchal C, Perret G, Richardet JP, Raffoux C, et al. High prevalence of diabetes mellitus in patients with chronic hepatitis C: a case-control study. Gastroenterol Clin Biol. 1996;20:544-8.

37. Knobler H, Schihmanter R, Zifroni A, Fenakel G, Schattner A. Increased risk of type 2 diabetes in noncirrhotic patients with chronic hepatitis $\mathrm{C}$ virus infection. Mayo Clin Proc. 2000;75:355-9. doi: 10.4065/75.4.355.

38. Thuluvath PJ, John PR. Association between hepatitis C, diabetes mellitus, and race: a case-control study. Am J Gastroenterol. 2003;98:438-41.

39. Mehta SH, Moore RD, Thomas DL, Chaisson RE, Sulkowski MS. The effect of HAART and HCV infection on the development of hyperglycemia among HIV-infected persons. J Acquir Immune Defic Syndr. 2003;33:577-84. doi: 10.1097/00126334-200308150-00005.

40. Hui JM, Sud A, Farrell GC, Bandara P, Byth K, Kench JG, et al. Insulin resistance is associated with chronic hepatitis $\mathrm{C}$ virus infection and fibrosis progression. Gastroenterology. 2003;125:1695-704. doi: 10.1053/j.gastro.2003.08.032.

41. Cosio FG, Pesavento TE, Osei K, Henry ML, Ferguson RM. Post-transplant diabetes mellitus: increasing incidence in renal allograft recipients transplanted in recent years. Kidney Int. 2001;59:732-7. doi: 10.1046/j.15231755.2001.059002732.x.

42. Baid S, Cosimi AB, Farrell ML, Schoenfeld DA, Feng S, Chung RT, et al. Posttransplant diabetes mellitus in liver transplant recipients: risk factors, temporal relationship with hepatitis $C$ virus allograft hepatitis, and impact on mortality. Transplantation. 2001;72:1066-72. doi: 10.1097/00007890-200109270-00015.

43. Abbott KC, Lentine KL, Bucci JR, Agodoa LY, Koff JM, Holtzmuller KC, et al. Impact of diabetes and hepatitis after kidney transplantation on patients who are affected by hepatitis C virus. J Am Soc Nephrol. 2004;15:3166-74. doi: 10.1097/01.ASN.0000145439.48387.BF.
44. Lecube A, Hernandez C, Genesca J, Esteban JI, Jardi R, Simo $\mathrm{R}$. High prevalence of glucose abnormalities in patients with hepatitis $\mathrm{C}$ virus infection: a multivariate analysis considering the liver injury. Diabetes Care. 2004;27:1171-5. doi: 10.2337/diacare.27.5.1171.

45. American Diabetes Association. 2. Classification and Diagnosis of Diabetes: Standards of Medical Care in Diabetes-2019. Diabetes Care. 2019;42(Suppl 1):S13-S28. doi: 10.2337/dc19-S002.

46. Masini M, Campani D, Boggi U, Menicagli M, Funel N, Pollera $\mathrm{M}$, et al. Hepatitis $\mathrm{C}$ virus infection and human pancreatic beta-cell dysfunction. Diabetes Care. 2005;28:940-1. doi: 10.2337/diacare.28.4.940.

47. Shintani Y, Fujie H, Miyoshi H, Tsutsumi T, Tsukamoto $\mathrm{K}$, Kimura $\mathrm{S}$, et al. Hepatitis $\mathrm{C}$ virus infection and diabetes: direct involvement of the virus in the development of insulin resistance. Gastroenterology. 2004;126:840-8. doi: 10.1053/j.gastro.2003.11.056.

48. Bureau C, Bernad J, Chaouche N, Orfila C, Béraud M, Gonindard C, et al. Nonstructural 3 protein of hepatitis $C$ virus triggers an oxidative burst in human monocytes via activation of NADPH oxidase. J Biol Chem. 2001;276:2307783. doi: 10.1074/jbc.M100698200.

49. Banerjee S, Saito K, Ait-Goughoulte M, Meyer K, Ray RB, Ray R. Hepatitis $C$ virus core protein upregulates serine phosphorylation of insulin receptor substrate- 1 and impairs the downstream akt/protein kinase B signaling pathway for insulin resistance. J Virol. 2008;82:2606-12. doi: 10.1128/ JVI.01672-07.

50. Burén J, Liu HX, Jensen J, Eriksson JW. Dexamethasone impairs insulin signalling and glucose transport by depletion of insulin receptor substrate-1, phosphatidylinositol 3-kinase and protein kinase B in primary cultured rat adipocytes. Eur J Endocrinol. 2002;146:419-29. doi: 10.1530/ eje.0.1460419.

51. Negro F. Mechanisms of hepatitis C virus-related insulin resistance. Clin Res Hepatol Gastroenterol. 2011;35:35863. doi: 10.1016/j.clinre.2011.01.011.

52. Gastaldi G, Goossens N, Clément S, Negro F. Current level of evidence on causal association between hepatitis $\mathrm{C}$ virus and type 2 diabetes: A review. J Adv Res. 2017;8:149-59. doi: 10.1016/j.jare.2016.11.003.

53. Horner SM, Gale M Jr. Regulation of hepatic innate immunity by hepatitis C virus. Nat Med. 2013;19:879-88. doi: 10.1038/nm.3253.

54. Bastard JP, Maachi M, Van Nhieu JT, Jardel C, Bruckert E, Grimaldi A, et al. Adipose tissue IL-6 content correlates with resistance to insulin activation of glucose uptake both in vivo and in vitro. J Clin Endocrinol Metab. 2002;87:20849. doi: 10.1210/jcem.87.5.8450.

55. Nelson DR, Lim HL, Marousis CG, Fang JW, Davis GL, Shen L, et al. Activation of tumor necrosis factor-alpha system in chronic hepatitis C virus infection. Dig Dis Sci. 1997;42:2487-94. doi: 10.1023/A:1018804426724. 
56. Krebs DL, Hilton DJ. SOCS proteins: negative regulators of cytokine signaling. Stem Cells 2001;19:378-87. doi: 10.1634/stemcells.19-5-378.

57. Oncül O, Top C, Cavuplu T. Correlation of serum leptin levels with insulin sensitivity in patients with chronic hepatitis-C infection. Diabetes Care 2002; 25: 937. doi: 10.2337/ diacare.25.5.937.

58. Mankouri J, Tedbury PR, Gretton S, Hughes ME, Griffin $\mathrm{SD}$, Dallas ML, et al. Enhanced hepatitis $\mathrm{C}$ virus genome replication and lipid accumulation mediated by inhibition of AMP-activated protein kinase. Proc Natl Acad Sci USA. 2010;107:11549-11554. doi: 10.1073/pnas.0912426107.

59. Cheung AT, Wang J, Ree D, Kolls JK, Bryer-Ash M. Tumor necrosis factor-alpha induces hepatic insulin resistance in obese Zucker $(\mathrm{fa} / \mathrm{fa}$ ) rats via interaction of leukocyte antigen-related tyrosine phosphatase with focal adhesion kinase. Diabetes 2000; 49: 810-9. doi: 10.2337/diabetes.49.5.810.

60. Ruan H, Lodish HF. Insulin resistance in adipose tissue: direct and indirect effects of tumor necrosis factor-alpha. Cytokine Growth Factor Rev. 2003;14:447-55. doi: 10.1016/S1359-6101(03)00052-2.

61. Fallahi P, Ferri C, Ferrari SM, Corrado A, Sansonno D, Antonelli A. Cytokines and HCV-related disorders. Clin Dev Immunol. 2012;2012:468107. doi: 10.1155/2012/468107.

62. Antonelli A, Ferri C, Fallahi P, Ferrari SM, Sebastiani M, Ferrari D, et al. High values of CXCL10 serum levels in mixed cryoglobulinemia associated with hepatitis $\mathrm{C}$ infection. Am J Gastroenterol. 2008;103:2488-94. doi: 10.1111/j.15720241.2008.02040.x.

63. Eslam M, Booth DR, George J, Ahlenstiel G. Interaction of IFNL3 with insulin resistance, steatosis and lipid metabolism in chronic hepatitis C virus infection. World J Gastroenterol. 2013;19:7055-61. doi: 10.3748/wjg.v19.i41.7055.

64. Yan FM, Chen AS, Hao F, Zhao XP, Gu CH, Zhao LB, et al. Hepatitis $C$ virus may infect extrahepatic tissues in patients with hepatitis C. World J Gastroenterol. 2000;6:805-11. doi: 10.3748/wjg.v6.i6.805.

65. Antonelli A, Ferri C, Ferrari SM, Colaci M, Sansonno D, Fallahi P. Endocrine manifestations of hepatitis $C$ virus infection. Nat Clin Pract Endocrinol Metab. 2009;5:26-34. doi: $10.1038 /$ ncpendmet 1027 .

66. Bogdanos DP, Rigopoulou EI. Viral/self-mimicry and immunological cross-reactivity as a trigger of hepatic $\mathrm{C}$ virus associated autoimmune diabetes. Diabetes Res Clin Pract. 2007;77:155-6. doi: 10.1016/j.diabres.2006.10.012.

67. Nakanishi K, Yoshimoto T, Tsutsui H, Okamura H. Interleukin-18 is a unique cytokine that stimulates both Th1 and Th2 responses depending on its cytokine milieu. Cytokine Growth Factor Rev. 2001;12:53-72. doi: 10.1016/ S1359-6101(00)00015-0.

68. Yumoto E, Higashi T, Nouso K, Nakatsukasa H, Fujiwara $\mathrm{K}$, Hanafusa $\mathrm{T}$, et al. Serum gamma-interferon-inducing factor (IL-18) and IL-10 levels in patients with acute hepatitis and fulminant hepatic failure. J Gastroenterol Hepatol. 2002;17:285-94. doi: 10.1046/j.1440-1746.2002.02690.x.
69. Lee LF, Xu B, Michie SA, Beilhack GF, Warganich T, Turley $\mathrm{S}$, et al. The role of TNF-alpha in the pathogenesis of type 1 diabetes in the nonobese diabetic mouse: analysis of dendritic cell maturation. Proc Natl Acad Sci USA. 2005;102:15995-6000. doi: 10.1073/pnas.0508122102.

70. Lecube A, Hernandez C, Genesca J, Esteban JI, Jardi R, Garcia $\mathrm{L}$, et al. Diabetes is the main factor accounting for the high ferritin levels detected in chronic hepatitis $\mathrm{C}$ virus infection. Diabetes Care. 2004;27:2669-75. doi: 10.2337/ diacare.27.11.2669.

71. Ramalho F. Hepatitis $\mathrm{C}$ virus infection and liver steatosis. Antiviral Res. 2003;60:125-7. doi: 10.1016/j.antiviral.2003.08.007.

72. Tsochatzis E, Papatheodoridis GV, Manesis EK, Chrysanthos N, Kafiri G, Petraki K, et al. Hepatic steatosis in genotype 4 chronic hepatitis $\mathrm{C}$ is mainly because of metabolic factors. Am J Gastroenterol. 2007;102:634-41. doi: 10.1111/j.1572-0241.2006.01025.x.

73. Schreuder TC, Gelderblom HC, Weegink CJ, Hamann D, Reesink HW, Devries JH, et al. High incidence of type 1 diabetes mellitus during or shortly after treatment with pegylated interferon alpha for chronic hepatitis $\mathrm{C}$ virus infection. Liver Int. 2008;28:39-46. doi: 10.1111/j.14783231.2007.01610.x.

74. Betterle C, Fabris P, Zanchetta R, Pedini B, Tositti G, Bosi $\mathrm{E}$, et al. Autoimmunity against pancreatic islets and other tissues before and after interferon-alpha therapy in patients with hepatitis $\mathrm{C}$ virus chronic infection. Diabetes Care. 2000;23:1177-181. doi: 10.2337/diacare.23.8.1177.

75. Chakrabarti D, Hultgren B, Stewart TA. IFN-alpha induces autoimmune $\mathrm{T}$ cells through the induction of intracellular adhesion molecule-1 and B7.2. J Immunol. 1996; 157:522-8.

76. Romero-Gómez M, Del Mar Viloria M, Andrade RJ, Salmerón J, Diago M, Fernández-Rodríguez CM, et al. Insulin resistance impairs sustained response rate to peginterferon plus ribavirin in chronic hepatitis $\mathrm{C}$ patients. Gastroenterology. 2005;128:636-41. doi: 10.1053/j.gastro.2004.12.049.

77. Alexia C, Fallot G, Lasfer M, Schweizer-Groyer G, Groyer A. An evaluation of the role of insulin-like growth factors (IGF) and of type-I IGF receptor signalling in hepatocarcinogenesis and in the resistance of hepatocarcinoma cells against drug induced apoptosis. Biochem Pharmacol. 2004;68:1003-15. doi: 10.1016/j.bcp.2004.05.029.

78. StuverSO, KuperH, TzonouA, Lagiou P, SpanosE,Hsieh CC, et al. Insulin-like growth factor 1 in hepatocellular carcinoma and metastaticliver cancer in men. Int J Cancer. 2000;87:11821. doi: 10.1002/1097-0215(20000701)87:1<118::aidijc17>3.0.co;2-w.

79. Dyal KH, Aguilar M, Bartos G, Holt WE, Bhuket T, Liu B, et al. Diabetes Mellitus increases risk of hepatocellular carcinoma in chronic hepatitis $\mathrm{C}$ virus patients: a systematic review. Dig Dis Sci. 2016;61:636-45. doi: 10.1007/s10620015-3983-3.

80. Arase Y, Kobayashi M, Suzuki F, Suzuki Y, Kawamura Y, Akuta N, et al. Effect of type 2 diabetes on risk for malignan- 
cies includes hepatocellular carcinoma in chronic hepatitis C. Hepatology. 2013;57:964-73. doi: 10.1002/hep.26087.

81. Ikeda A, Ikeda K, Takai A, Takahashi K, Ueda Y, Marusawa $\mathrm{H}$, et al. Hepatitis $\mathrm{C}$ treatment with sofosbuvir and ledipasvir accompanied by immediate improvement in hemoglobin A1C. Digestion. 2017;96:228-30. doi: 10.1159/000484237.

82. Adinolfi LE, Nevola R, Guerrera B, D’Alterio G, Marrone A, Giordano $\mathrm{M}$, et al. Hepatitis $\mathrm{C}$ virus clearance by directacting antiviral treatments and impact on insulin resistance in chronic hepatitis C patients. J Gastroenterol Hepatol. 2018;33:1379-82. doi: 10.1111/jgh.14067.

83. Ciancio A, Bosio R, Bo S, Pellegrini M, Sacco M, Vogliotti E, et al. Significant improvement of glycemic control in diabetes patients with $\mathrm{HCV}$ infection responding to direct-acting antiviral agents. J Med Virol. 2018;90:320-7. doi: 10.1002/ jmv.24954.

84. Dawood AA, Nooh MZ, Elgamal AA. Factors associated with improved glycemic control by direct-acting antiviral agent treatment in Egyptian type 2 diabetes mellitus patients with chronic hepatitis $\mathrm{C}$ genotype 4. Diabetes Metab J. 2017;41:316-21. doi: 10.4093/dmj.2017.41.4.316.

85. Abdel-Hamid N, Jubori TA, Farhan A, Mahrous M, Gouri A, Awad E, et al. Underlying pathways for interferon risk to type II diabetes mellitus. Curr Diabetes Rev. 2013;9(6):472-7.

86. Li J, Zhang T, Gordon SC, Rupp LB, Trudeau S, Holmberg $\mathrm{SD}$, et al. Impact of sustained virologic response on risk of type 2 diabetes among hepatitis $\mathrm{C}$ patients in the United States. J Viral Hepat. 2018;25(8):952-958. doi: 10.1111/ jvh.12887.

87. Li J, Gordon SC, Rupp LB, Zhang T, Trudeau S, Holmberg $\mathrm{SD}$, et al. Sustained virological response to hepatitis C treatment decreases the incidence of complications associated with type 2 diabetes. Aliment Pharmacol Ther. 2019;49:599-608. doi: 10.1111/apt.15102.

88. Gilad A, Fricker ZP, Hsieh A, Thomas DD, Zahorian T, Nunes DP. Sustained Improvement in Type 2
Diabetes Mellitus is Common After Treatment of Hepatitis C Virus With Direct-acting Antiviral Therapy. J Clin Gastroenterol. 2019;53(8):616-20. doi: 10.1097/ MCG.0000000000001168.

89. Hum J, Jou JH, Green PK, Berry K, Lundblad J, Hettinger $\mathrm{BD}$, et al. Improvement in glycemic control of type 2 diabetes after successful treatment of hepatitis $\mathrm{C}$ virus. Diabetes Care. 2017;40:1173-80. doi: 10.2337/dc17-0485.

90. Crook ED, Penumalee S, Gavini B, Filippova K. Hepatitis $\mathrm{C}$ is a predictor of poorer renal survival in diabetic patients. Diabetes Care 2005;28:2187-91. doi: 10.2337/diacare.28.9.2187.

91. Walsh MJ, Jonsson JR, Richardson MM, Lipka GM, Purdie $\mathrm{DM}$, Clouston $\mathrm{AD}$, et al. Non-response to antiviral therapy is associated with obesity and increased hepatic expression of suppressor of cytokine signalling 3 (SOCS-3) in patients with chronic hepatitis C, viral genotype 1. Gut. 2006;55:529-35. doi: 10.1136/gut.2005.069674.

92. Sanyal AJ. Role of insulin resistance and hepatic steatosis in the progression of fibrosis and response to treatment in hepatitis C. Liver Int. 2011;31(Suppl 1):23-8. doi: 10.1111/j.1478-3231.2010.02397.x.

93. Shaw RJ, Lamia KA, Vasquez D, Koo SH, Bardeesy N, Depinho RA, et al. The kinase LKB1 mediates glucose homeostasis in liver and therapeutic effects of metformin. Science. 2005;310:1642-6. doi: 10.1126/science.1120781.

94. O’Leary JG, Chan JL, McMahon CM, Chung RT. Atorvastatin does not exhibit antiviral activity against $\mathrm{HCV}$ at conventional doses: a pilot clinical trial. Hepatology. 2007;45:895-8. doi: 10.1002/hep.21554.

95. Wang CS, Yao WJ, Chang TT, Wang ST, Chou P. The impact of type 2 diabetes on the development of hepatocellular carcinoma in different viral hepatitis statuses. Cancer Epidemiol Biomarkers Prev. 2009; 18:2054-60. doi: 10.1158/10559965.EPI-08-1131. 